\title{
Enteric Fever Caused by Salmonella enterica Serovars with Reduced Susceptibility of Fluoroquinolones at a Community Based Teaching Hospital of Nepal
}

\author{
Anjeela Bhetwal, ${ }^{1}$ Anjila Maharjan, ${ }^{1}$ Puspa Raj Khanal, ${ }^{1}$ and Narayan Prasad Parajuli ${ }^{1,2}$ \\ ${ }^{1}$ Department of Laboratory Medicine, Manmohan Memorial Institute of Health Sciences, Kathmandu, Nepal \\ ${ }^{2}$ Division of Clinical Microbiology, Department of Clinical Laboratory Services, Manmohan Memorial Medical College and \\ Teaching Hospital, Kathmandu, Nepal
}

Correspondence should be addressed to Anjeela Bhetwal; anjeela065@gmail.com

Received 10 July 2017; Revised 7 October 2017; Accepted 18 October 2017; Published 6 November 2017

Academic Editor: Karl Drlica

Copyright (C) 2017 Anjeela Bhetwal et al. This is an open access article distributed under the Creative Commons Attribution License, which permits unrestricted use, distribution, and reproduction in any medium, provided the original work is properly cited.

\begin{abstract}
Enteric fever continues to be an important public health problem especially in developing countries of the tropical region including Nepal. In this study, we aimed to investigate the incidence of enteric fever associated with Salmonella enterica and determine its antimicrobial susceptibilities to therapeutic antimicrobials in a community based teaching hospital of Nepal. A total of 2,304 blood samples from suspected enteric fever patients attending Manmohan Memorial Teaching Hospital were processed with standard microbiological methods for the isolation and identification of bacterial pathogens. The Salmonella enterica clinical strains were subjected to antimicrobial susceptibility testing by Kirby-Bauer disk diffusion method, and the results were interpreted according to the criteria suggested by the Clinical and Laboratory Standards Institute (CLSI). A total of 245 (10.6\%) cases of enteric fever associated with Salmonella enterica were confirmed by blood culture. Out of them, 162 (66.1\%) were caused by Salmonella Typhi and 83 (33.9\%) by Salmonella Paratyphi. On Kirby-Bauer disk diffusion antimicrobial susceptibility testing, Salmonella isolates were highly susceptible to cefixime (100\%), ceftriaxone (100\%), ampicillin (97.9\%), cotrimoxazole (94.6\%), azithromycin (96.7\%), tetracycline (95.5\%), and chloramphenicol (97.5\%), respectively. Two hundred twenty-six (92.2\%) of Salmonella isolates were nalidixic acid resistant with reduced susceptibility to ciprofloxacin (36.7\%) and ofloxacin (54.8\%), respectively. Although the rate of MDR Salmonella strains was very low $(<5 \%)$, their reduced susceptibility to fluoroquinolones has restricted their routine empirical use. Third generation cephalosporins are the safest choice for empirical use but ampicillin, cotrimoxazole, azithromycin, and chloramphenicol can be effective alternatives.
\end{abstract}

\section{Background}

Enteric fever is a life-threatening systemic illness caused by serovars of human-adapted pathogen, Salmonella enterica [1]. It is an acute and invasive infection of the gastrointestinal system and causes a devastating burden in many low- and middle-income countries with significant morbidity and mortality $[2,3]$. The recent global incidence of enteric fever has been reported to range from 11.9 million to 26.9 million per year with approximate case fatality rate of $1 \%$ $[4,5]$. In Nepal, enteric fever or typhoid fever, commonly known as "Bisham Jwaro" (fever with poison), is prevalent in mountains, valleys, and southern Terai region as an endemic disease, with its peak incidence occurring from May to August. It is one of the leading diagnoses of fever in most of the hospitals in Nepal. Series of enteric fever outbreaks with variable drug susceptibilities have been reported from the country, and the vast bulk of them have been linked to fecal contamination of foods and drinking water $[6,7]$.

Antimicrobial agents are the mainstay of therapy in enteric fever so as to prevent the complications associated with severe illness and death of the patients [8]. However, the reduced susceptibility of Salmonella enterica isolates to commonly used antibiotics continues to be a major problem for effective therapy of enteric fever, particularly in developing countries $[9,10]$. Multidrug resistant Salmonella enterica 
strains (resistant to chloramphenicol, ampicillin, and cotrimoxazole) are increasingly reported from Asian countries $[11,12]$. In Nepal, also, there have been several enteric fever epidemics with changing antibiotic resistance patterns $[13,14]$ since the first report of multiple drug resistant (MDR) $S$. Typhi in 1991 [15]. Due to the emergence of MDR strains, the use of chloramphenicol, ampicillin, and cotrimoxazole has become infrequent and quinolones became the first choice for the treatment of typhoid fever in endemic areas [16]. Subsequently, during the last few years, nalidixic acid resistant strains associated with reduced susceptibility to fluoroquinolones in the patients treated with quinolones have been increasingly reported elsewhere [17] including Nepal $[6,18]$. Cephalosporins and macrolides are nowadays the therapeutic choices for enteric fever cases in our region [19], but the emergence of multidrug resistant and extended spectrum $\beta$-lactamase (ESBL) producing strains has created a therapeutic challenge [14].

In this backdrop, when the treatment options for enteric fever are decreasing, extensive workup and evaluation of alternative choices for effective therapy and management of enteric fever cases are becoming vital. Therefore, this study was intended to determine the spectrum of Salmonella enterica serovars isolated from the blood culture of the patients suffering from enteric fever and their antibiotic susceptibility pattern to commonly used antibiotics in a community based tertiary care teaching hospital in Kathmandu, Nepal.

\section{Methodology}

2.1. Study Design and Setting. This was a laboratory-based descriptive study carried out over a period of two years (April 2015 to March 2017) in the Department of Clinical Microbiology of Manmohan Memorial Teaching Hospital (MMTH), a community based tertiary care teaching hospital with 300patient beds in Swayambhu, Kathmandu, Nepal. The hospital is located outside the main city of Kathmandu and is a major hospital for rural villages nearby capital.

2.2. Inclusion and Exclusion Criteria. In this study, patients clinically suspected of enteric fever presented to our hospital were enrolled. However, the patients already on antibiotics and repeated samples from the same patient were excluded.

2.3. Specimen Collection, Processing, and Identification of Salmonella Isolates. Patients visiting outpatient departments or admitted to the inpatient units suspected of enteric fever were investigated clinically by respective unit physicians. A blood culture specimen was taken with the aseptic technique by cleansing of the collection site with $70 \%$ alcohol and subsequently followed by povidone iodine. Five milliliters (for pediatric patients) and $10 \mathrm{ml}$ (for adult patients) of blood specimen were collected and inoculated into brain heart infusion (BHI) broth at the blood to broth ratio of $1: 10$. After incubation, at $37^{\circ} \mathrm{C}$ for 24,48 , and 72 hours, subculture was made on blood agar and MacConkey agar plates regardless of the turbidity. The plates were observed for bacterial growth after $24 \mathrm{hrs}$ of aerobic incubation at $37^{\circ} \mathrm{C}$. Salmonella enterica isolates were identified using standard microbiological techniques: biotyping (colony morphology, staining reaction, and biochemical characteristics) and serotyping using specific antisera (Denka Seiken Co. Ltd., Tokyo, Japan) [20]. Samples were considered sterile if no growth was observed on subculture after 7 days of aerobic incubation at $37^{\circ} \mathrm{C}$. Patient information, that is, patient name, age, sex, ward/bed number (if admitted), brief clinical history, duration of hospital stay, and history of antibiotic use, was taken.

2.4. Antimicrobial Susceptibility Testing. Antimicrobial susceptibility testing of the isolated strains of Salmonella enterica was carried out using the disk diffusion method (modified Kirby-Bauer method) on Mueller-Hinton agar (HiMedia, India) following standard procedures recommended by the Clinical and Laboratory Standards Institute (CLSI), Wayne, USA [21]. We analyzed the susceptibility of common therapeutic antimicrobial agents including ampicillin $(10 \mu \mathrm{g})$, nalidixic acid $(30 \mu \mathrm{g})$, ofloxacin $(5 \mu \mathrm{g})$, ciprofloxacin $(5 \mu \mathrm{g})$, chloramphenicol $(30 \mu \mathrm{g})$, cotrimoxazole $(25 \mu \mathrm{g})$, cefixime $(30 \mu \mathrm{g})$, ceftriaxone $(30 \mu \mathrm{g})$, cefotaxime $(30 \mu \mathrm{g})$, azithromycin $(15 \mu \mathrm{g})$, and tetracycline $(30 \mu \mathrm{g})$ (HiMedia Laboratories, India). The results of the antibiotic susceptibility were determined on the basis of interpretative zone diameters suggested by CLSI [21]. For standardization, Escherichia coli ATCC-25922 was used as the control organism for antibiotic sensitivity.

2.5. Statistical Analysis of Data. Data regarding the bacterial isolates, their susceptibility to various antibiotics, and other information were entered and analyzed using the Statistical Package for Social Sciences (SPSS ${ }^{\mathrm{TM}}$ ) version 20.0 (IBM, Armonk, NY, USA). The results are presented in percentagebased distribution.

2.6. Ethical Approval. Written approval (Ref 009/MMIHS/ 2072) was obtained from Institutional Review Committee of Manmohan Memorial Institute of Health Sciences (MMIHS) before starting the study. Written informed consent was taken from every patient or their guardians before enrollment into the study.

\section{Results}

3.1. Patients with Enteric Fever and Their Demographics. During the study period, out of a total of 2,304 blood culture specimens from the patients suspected with enteric fever, 245 (10.63\%) were found positive for the growth of Salmonella enterica, confirming the enteric fever. More samples were from outpatients $(1701,73.8 \%)$ as compared to inpatients $(603,26.2 \%)$. Male patients $(149,60.8 \%)$ constitute the major subgroup affected with enteric fever, and Salmonella enterica serovar Typhi $(162,66.1 \%)$ was the common serovar associated with enteric fever in our setting. In addition, the proportion $(178,72.6 \%)$ of enteric fever cases occurred in patients of the age group of 15-44 years was higher than any other age group (Table 1). 
TABLE 1: Patients with enteric fever and their demographics.

\begin{tabular}{lcccc}
\hline Age group (years) & Total (\%) & Confirmed (\%) & $\begin{array}{c}\text { Enteric fever cases } \\
\text { Salmonella Typhi (\%) }\end{array}$ & Salmonella Paratyphi (\%) \\
\hline$<5$ & $261(11.3)$ & $17(6.5)$ & $11(64.7)$ & $6(35.3)$ \\
$5-14$ & $355(15.4)$ & $37(10.4)$ & $22(59.4)$ & $15(40.6)$ \\
$15-44$ & $1,210(52.2)$ & $178(14.7)$ & $119(66.8)$ & $59(33.2)$ \\
$45-59$ & $281(12.2)$ & $8(2.9)$ & $6(75.0)$ & $2(25.0)$ \\
$\geq 60$ & $197(8.5)$ & $5(2.5)$ & $4(80.0)$ & $1(20.0)$ \\
\hline Total & 2,304 & $245(10.6)$ & $162(66.1)$ & $83(33.9)$ \\
\hline
\end{tabular}

TABLE 2: Antimicrobial susceptibilities of Salmonella enterica serovars.

\begin{tabular}{|c|c|c|c|c|c|c|}
\hline \multirow{2}{*}{ Antibiotics } & \multicolumn{3}{|c|}{ Salmonella enterica serovar Typhi $(n=162)$} & \multicolumn{3}{|c|}{ Salmonella enterica serovar Paratyphi $(n=83)$} \\
\hline & $S(\%)$ & $I(\%)$ & $R(\%)$ & $S(\%)$ & $I(\%)$ & $R(\%)$ \\
\hline Ampicillin & $158(97.6)$ & $0(0)$ & $4(2.4)$ & $82(98.8)$ & $0(0)$ & $1(1.2)$ \\
\hline Nalidixic acid & $17(10.5)$ & $0(0)$ & $145(89.5)$ & $2(2.4)$ & $0(0)$ & $81(97.6)$ \\
\hline Ciprofloxacin & $63(39.0)$ & $95(58.6)$ & $4(2.4)$ & $39(46.9)$ & $35(42.2)$ & $9(10.9)$ \\
\hline Ofloxacin & $89(55.0)$ & $65(40.0)$ & $8(5.0)$ & $54(65.0)$ & $22(26.6)$ & $7(8.4)$ \\
\hline Cotrimoxazole & $151(93.2)$ & $0(0)$ & $11(6.8)$ & $81(97.6)$ & $0(0)$ & $2(2.4)$ \\
\hline Cefixime & $162(100)$ & $0(0)$ & $0(0)$ & $83(100)$ & $0(0)$ & $0(0)$ \\
\hline Cefotaxime & $162(100)$ & $0(0)$ & $0(0)$ & $83(100)$ & $0(0)$ & $0(0)$ \\
\hline Ceftriaxone & $162(100)$ & $0(0)$ & $0(0)$ & $83(100)$ & $0(0)$ & $0(0)$ \\
\hline Azithromycin & $156(96.3)$ & $0(0)$ & $6(3.7)$ & $81(97.6)$ & $0(0)$ & $2(2.4)$ \\
\hline Tetracycline & $154(95.1)$ & $0(0)$ & $8(4.9)$ & $80(96.3)$ & $0(0)$ & $3(3.7)$ \\
\hline Chloramphenicol & $156(96.3)$ & $0(0)$ & $6(3.7)$ & $83(100)$ & $0(0)$ & $0(0)$ \\
\hline
\end{tabular}

$S$ : sensitive, $I$ : intermediate sensitive, $R$ : resistant.

3.2. Antimicrobial Susceptibilities of Salmonella Serovars Typhi and Paratyphi. Table 2 illustrates the susceptibilities of Salmonella enterica serovars Typhi and Paratyphi in our study. Overall, $39.0 \%$ and $46.9 \%$ of Salmonella Typhi and Paratyphi serovars were susceptible to ciprofloxacin, while $55.0 \%$ and $65.0 \%$ of them were susceptible to ofloxacin. Reduced susceptibility to ciprofloxacin $(2.4 \%$ and $10.9 \%$ resistant, $58.6 \%$ and $42.2 \%$ intermediate susceptible to Typhi and Paratyphi, resp.) and ofloxacin (5.0\% and $8.4 \%$ resistant, $40.0 \%$ and $26.6 \%$ intermediate susceptible to Typhi and Paratyphi, resp.) was observed. Other than fluoroquinolones, the overall susceptibility of Salmonella isolates to chloramphenicol, ampicillin, cotrimoxazole, and azithromycin was found to be excellent, that is, $97.5 \%, 97.9 \%, 94.6 \%$, and $96.7 \%$ each, suggesting revival of conventional antibiotics in our setting.

3.3. Nalidixic Acid Resistant S. Typhi and S. Paratyphi. Nalidixic acid resistance was very much common among the isolates of Salmonella enterica. Overall, $92.2 \%$ of the isolates were NA resistant. Although statistically nonsignificant $(p>$ $0.05), S$. Paratyphi strains showed higher rate (97.6\%) of NAR than S. Typhi (89.5\%) (Table 3).

\section{Discussion}

Enteric fever remains a major cause of febrile illness in the urban areas of endemic countries with limited water and
TABLE 3: Distribution of nalidixic acid (NA) resistant $S$. Typhi and S. Paratyphi.

\begin{tabular}{|c|c|c|c|}
\hline \multirow{2}{*}{ Serovars } & \multicolumn{2}{|c|}{ Nalidixic acid } & \multirow{2}{*}{$p$} \\
\hline & Resistant (\%) & Sensitive (\%) & \\
\hline S. Typhi & $145(89.5)$ & $17(10.5)$ & \multirow{3}{*}{0.624} \\
\hline S. Paratyphi & $81(97.6)$ & $2(2.4)$ & \\
\hline Total & $226(92.2)$ & $19(7.8)$ & \\
\hline
\end{tabular}

sanitation infrastructure [22]. World Health Organization (WHO) has recommended vaccination with existing $\mathrm{Vi}$ polysaccharide vaccine targeting high-risk areas of typhoid fever [16]. Besides, estimation of the disease burden and its etiology along with antimicrobial susceptibilities would be helpful in the development of effective prevention and control interventions. Nepal is a pocket area of typhoid endemicity due to the poor sanitation status and cross-contamination of food and drinking water with sewage [7].

Overall, the incidence rate of enteric fever caused by serovars of Salmonella enterica in our hospital was $10.6 \%$. The finding of our study is similar to the reports of Sharma et al. (8.9\%) [23], Shrestha et al. (13.3\%) [24], and Easow et al. (15.6\%) [25] from nearby hospitals of Nepal. The lower rates of blood-culture-positive enteric fever might also be due to the use of antibiotics prior to the blood culture and low blood volume used for culture $(10 \mathrm{ml}$ for adult and $5 \mathrm{ml}$ 
for children) as well as self-medication before arrival to the hospital. However, we did not evaluate the prior antibiotic consumption by the patients before enrollment. In addition, our data is well supported by recent epidemiological studies in Nepal $[26,27]$, where the great bulk of undifferentiated febrile illness was associated with other atypical organisms [27]. In addition, male patients $(149,12.5 \%)$ and patients in the 15-44 age group $(178,72.6 \%)$ showed higher incidence of enteric fever cases, corroborating the previous reports from Nepal $[19,28]$. Probable reason behind this variation might be regular eating-out habit of males and the adult age population consuming infected food and water from restaurants.

Out of 245 culture-confirmed enteric fever cases, 162 (66.1\%) were caused by Salmonella Typhi and 83 (33.9\%) were by Salmonella Paratyphi. The dominance of Typhi serovars in enteric fever in our study complies with the observation made by Adhikari et al. (64.1\% and $35.9 \%$ of the S. Typhi and $S$. Paratyphi, resp.) [28]. However, Shirakawa et al. documented $S$. Paratyphi as more prevalent serovar in Kathmandu, Nepal [18], which is supported by another recent study of Pramod et al. (35.9\% S. Typhi and 64.1\% S. Paratyphi) [29]. Although, there is no such well-established cause for serovar variation in enteric fever cases, higher incidence of Typhi might be due to waterborne transmission of $S$. Typhi as it usually involves smaller inocula than paratyphoid achieved through food borne transmission that requires large inocula [30].

Fluoroquinolones (FQs), ciprofloxacin and ofloxacin, are the mainstay of therapy against Salmonella infections because they are available for oral use and are also less expensive options [31]. However, they are increasingly becoming ineffective in enteric fever cases due to the emergence of nalidixic acid resistant (NAR) strains $[12,13]$. In our study, rate of NAR, a phenotypic marker for reduced susceptibility to fluoroquinolones, was very high (92.2\%). Moreover, S. Paratyphi strains showed even higher rate $(97.6 \%)$ of NAR than S. Typhi (89.5\%). Similar rates of resistance to nalidixic acid (NA) among $S$. Paratyphi isolates were reported in the studies of Acharya et al. [30], Shirakawa et al. [18], and Chand et al. [19] from Nepal. The rate of resistance to NA we found is high when compared to the reports of Shrestha et al. (83.1\%) [24] from Nepal and Kadhiravan et al. from India $(78 \%)$ [32]. The high rate of resistance to NA and emergence of strains with full resistance to FQs by Salmonella spp constitute a major problem in Nepal [13]. On the other hand, the main cause of resistance to quinolones in Gramnegative bacteria, including Salmonella, is the mutation in the genes coding for DNA gyrase (gyrA and gyrB) and topoisomerase IV (parC and parE) [33]. Enhanced active efflux and early overproduction of the AcrA pump in isolates with the gyrA mutation could be responsible for the decrease in susceptibility to FQs. Low level of resistance to ciprofloxacin, probably due to the point mutation in the gyrA gene, may not be detected by in vitro susceptibility tests using the current MIC breakpoints for ciprofloxacin. Therefore, in vitro resistance to NA can be used to detect this low level resistance $[31,34]$. However, susceptibility testing generally adopted in the resource-poor laboratories of developing countries including Nepal is limited to disk diffusion technique which may not be adequate to determine reduced susceptibility to FQs $[12,24]$.
Furthermore, the increasing number of isolates having reduced susceptibility to ciprofloxacin and ofloxacin may be due to irrational use of FQs, as the empirical antimicrobial agent, and emergence of NAR strains [31]. Besides, for NAR strains, reduced susceptibility to ciprofloxacin $(31.7 \%$ and $45.6 \%$ susceptible, $65.6 \%$ and $43.3 \%$ intermediate susceptible, and $2.7 \%$ and $11.1 \%$ resistant in Typhi and Paratyphi, resp.) and ofloxacin $(49.7 \%$ and $64.2 \%$ susceptible, $44.8 \%$ and $27.1 \%$ intermediate susceptible, and $5.6 \%$ and $8.7 \%$ resistant in Typhi and Paratyphi, resp.) was found. In NAS Salmonella Typhi and Paratyphi isolates, sensitivity to fluoroquinolones reached $100 \%$. Reduced susceptibilities to fluoroquinolones were recorded previously for this region [28, 29, 35]. However, $100 \%$ susceptibility of Salmonella Typhi and $96.7 \%$ susceptibility of Salmonella Paratyphi to ciprofloxacin were observed in a study by Chand et al. [19]. In our study, the isolates with reduced fluoroquinolones susceptibility were also uniformly resistant to NA. NA susceptibility showed a predictive value of $100 \%$ for ciprofloxacin and ofloxacin susceptibility, whereas NA resistance showed a predictive value of $58.95 \%$ for ciprofloxacin and ofloxacin resistance.

Besides fluoroquinolones, the overall susceptibility of Salmonella isolates to chloramphenicol was found to be $97.5 \%$ (96.3\% for S. Typhi and 100\% for S. Paratyphi). Susceptibility of Salmonella isolates to other first-line drugs, that is, ampicillin, cotrimoxazole, and azithromycin, was also excellent, 97.9\%, 94.6\%, and 96.7\%, respectively. Chloramphenicol was once considered the drug of choice for enteric fever acquired resistance within few years of its introduction, but later, MDR Salmonella resistant to chloramphenicol, ampicillin, and trimethoprim sulfamethoxazole emerged in the late 1980 s and early 1990s [36, 37]. Reemergence of susceptibility to chloramphenicol and other first-line drugs in previously resistant areas has been reported in studies done earlier [19, $24,38-40]$. The decreased use of first-line antibiotics in treating Salmonella and other infections could be likely the reason for this reemergence of susceptibility [24]. Cephalosporins (ceftriaxone, cefotaxime, and cefixime) exhibited excellent efficacy towards isolated Salmonella serovars with 100\% sensitivity. Therefore, oral cephalosporins and macrolides are the first-line agents for empirical treatment of enteric fever cases $[4,41]$. Fluoroquinolones would still be the effective therapeutic regimen in our scenario because a good proportion of quinolones is found susceptible, but susceptibility test should be performed before starting the quinolone therapy.

4.1. Limitation of the Study. Although this was a hospitalbased study, we could not evaluate the risk factors and outcomes of enteric fever in our setting. Underequipped laboratories, lack of resources for molecular techniques, limited options for antimicrobial susceptibilities are the main drawbacks to high-quality data for clinical evaluation. This is a single-center study; multicenter cohort study including national wide geographical area would have generated more significant results in our country.

\section{Conclusion}

Sensitivity pattern of the Salmonella isolates is changing especially in the community settings with reemergence of 
ampicillin, cotrimoxazole, and chloramphenicol sensitive Salmonella strains. Fluoroquinolones are becoming less effective because of haphazard use and are no more suitable for empirical use in our settings. Use of different antibiotic agents as per the sensitivity patterns and disease severity can help to decrease excessive use of cephalosporin and macrolide and ultimately help to effectively delay the emergence of resistance to these agents. Only good foresight and proper antibiotic policy can save our communities from the inevitable burden of antibiotic resistance.

$\begin{array}{ll}\text { Abbreviations } \\ \text { ASM: } & \text { American Society for Microbiology } \\ \text { ATCC: } & \text { American type culture collection } \\ \text { BHI: } & \text { Brain heart infusion broth } \\ \text { CLSI: } & \text { Clinical and Laboratory Standard Institute } \\ \text { FQs: } & \text { Fluoroquinolones } \\ \text { MDR: } & \text { Multidrug resistant } \\ \text { NARS: } & \text { Nalidixic acid resistant Salmonella } \\ \text { S. Typhi: } & \text { Salmonella enterica subspecies enterica } \\ & \text { serovar Typhi } \\ \text { S. Paratyphi: } & \text { Salmonella enterica subspecies enterica } \\ & \text { serovar Paratyphi } \\ \text { ZOI: } & \text { Zone of inhibition. }\end{array}$

\section{Ethical Approval}

This research was approved by the Institutional Review Committee of Manmohan Memorial Institute of Health Sciences (IRC MMIHS), Kathmandu, Nepal. Letter of approval (Ref number 009/MMIHS/2072) was obtained after submitting and presenting the proposal to the committee.

\section{Disclosure}

Anjila Maharjan, Puspa Raj Khanal, and Narayan Prasad Parajuli are coauthors.

\section{Conflicts of Interest}

There are no conflicts of interest to disclose.

\section{Authors' Contributions}

Anjeela Bhetwal and Narayan Prasad Parajuli designed the study and reviewed the literature. Anjeela Bhetwal and Anjila Maharjan performed the laboratory investigations. Anjeela Bhetwal and Narayan Prasad Parajuli prepared the manuscript. All the authors contributed towards the preparation of the draft and the revision of the paper, gave final approval of the version to be published, and agree to be accountable for all aspects of the work.

\section{References}

[1] J. A. Crump and E. D. Mintz, "Global trends in typhoid and paratyphoid fever," Clinical Infectious Diseases, vol. 50, no. 2, pp. 241-246, 2010.
[2] J. Wain, R. S. Hendriksen, M. L. Mikoleit, K. H. Keddy, and R. L. Ochiai, “Typhoid fever," The Lancet, vol. 385, no. 9973, pp. 11361145, 2015.

[3] J. A. Crump, F. G. Youssef, S. P. Luby et al., "Estimating the incidence of typhoid fever and other febrile illnesses in developing countries," Emerging Infectious Diseases, vol. 9, no. 5, pp. 539-544, 2003.

[4] J. A. Crump, M. Sjölund-Karlsson, M. A. Gordon, and C. M. Parry, "Epidemiology, clinical presentation, laboratory diagnosis, antimicrobial resistance, and antimicrobial management of invasive Salmonella infections," Clinical Microbiology Reviews, vol. 28, no. 4, pp. 901-937, 2015.

[5] A. D. Steele, D. C. Hay Burgess, Z. Diaz, M. E. Carey, and A. K. M. Zaidi, "Challenges and Opportunities for Typhoid Fever Control: A Call for Coordinated Action," Clinical Infectious Diseases, vol. 62, pp. s4-s8, 2016.

[6] A. P. Maskey, B. Basnyat, G. E. Thwaites, J. I. Campbell, J. J. Farrar, and M. D. Zimmerman, "Emerging trends in enteric fever in Nepal: 9124 cases confirmed by blood culture 1993-2003," Transactions of the Royal Society of Tropical Medicine and Hygiene, vol. 102, no. 1, pp. 91-95, 2008.

[7] M. D. Lewis, O. Serichantalergs, C. Pitarangsi et al., "Typhoid fever: A massive, single-point source, multidrug-resistant outbreak in Nepal," Clinical Infectious Diseases, vol. 40, no. 4, pp. 554-561, 2005.

[8] R. C. Charles, T. Sultana, M. M. Alam et al., "Identification of Immunogenic Salmonella enterica Serotype Typhi Antigens Expressed in Chronic Biliary Carriers of S. Typhi in Kathmandu, Nepal," PLOS Neglected Tropical Diseases, vol. 7, no. 8, Article ID e2335, 2013.

[9] M. V. Jesudason and T. J. John, "Plasmid mediated multidrug resistance in Salmonella typhi," Indian Journal of Medical Research - Section A Infectious Diseases, vol. 95, pp. 66-67, 1992.

[10] T. Butt, R. N. Ahmad, A. Mahmood, and S. Zaidi, "Ciprofloxacin Treatment Failure in Typhoid Fever Case, Pakistan," Emerging Infectious Diseases, vol. 9, no. 12, pp. 1621-1622, 2003.

[11] S. Mohanty, K. Renuka, S. Sood, B. K. Das, and K. Kapil, "Antibiogram pattern and seasonality of Salmonella serotypes in a North Indian tertiary care hospital," Epidemiology and Infection, vol. 134, no. 5, pp. 961-966, 2006.

[12] G. A. Menezes, B. N. Harish, M. A. Khan, W. H. F. Goessens, and J. P. Hays, "Antimicrobial resistance trends in blood culture positive Salmonella Typhi isolates from Pondicherry, India, 2005-2009," Clinical Microbiology and Infection, vol. 18, no. 3, pp. 239-245, 2012.

[13] D. Acharya, S. Malla, D. R. Bhatta, and S. P. Dumre, "Multidrug resistant Salmonella enterica serovar typhi," Journal of Nepal Medical Association, vol. 48, no. 174, pp. 196-197, 2009.

[14] B. M. Pokharel, J. Koirala, R. K. Dahal, S. K. Mishra, P. K. Khadga, and N. R. Tuladhar, "Multidrug-resistant and extended-spectrum beta-lactamase (ESBL)-producing Salmonella enterica (serotypes Typhi and Paratyphi A) from blood isolates in Nepal: surveillance of resistance and a search for newer alternatives," International Journal of Infectious Diseases, vol. 10, no. 6, pp. 434-438, 2006.

[15] J. P. Watson, "Multi-Resistant Typhoid Fever in Nepal," Tropical Doctor, vol. 22, no. 4, p. 172, 1992.

[16] WHO: Background document: the diagnosis, treatment and prevention of typhoid fever. In, vol. WHO/V\&B/03.07. Geneva: World Health Organization, 2003.

[17] T. T. Chau, J. I. Campbell, and C. M. Galindo, "Antimicrobial drug resistance of Salmonella enterica serovar Typhi in Asia 
and molecular mechanism of reduced susceptibility to the fluoroquinolones," Antimicrobial Agents and Chemotherapy, vol. 51, no. 12, pp. 4315-4323, 2007.

[18] T. Shirakawa, B. Acharya, S. Kinoshita, S. Kumagai, A. Gotoh, and M. Kawabata, "Decreased susceptibility to fluoroquinolones and gyrA gene mutation in the Salmonella enterica serovar Typhi and Paratyphi A isolated in Katmandu, Nepal, in 2003," Diagnostic Microbiology And Infectious Disease, vol. 54, no. 4, pp. 299-303, 2006.

[19] H. J. Chand, K. R. Rijal, B. Neupane, V. K. Sharma, and B. Jha, "Re-emergence of susceptibility to conventional first line drugs in Salmonella isolates from enteric fever patients in Nepal," The Journal of Infection in Developing Countries, vol. 8, no. 11, pp. 1483-1487, 2014.

[20] H. D. Isenberg, Clinical Microbiology procedure handbook, vol. 1, 2nd edn. ASM Press, Washington DC: American Society for Microbiology, 2004.

[21] Performance Standards for Antimicrobial Disk Susceptibility Tests In, vol. M100-S25. Wayne, PA: Clinical and Laboratory Standards Institute, 2015.

[22] V. Mogasale, V. V. Mogasale, E. Ramani et al., "Revisiting typhoid fever surveillance in low and middle income countries: Lessons from systematic literature review of population-based longitudinal studies," BMC Infectious Diseases, vol. 16, no. 1, article no. 35, 2016.

[23] N. Sharma, R. Koju, B. Karmacharya et al., "Typhoid fever in Dhulikhel hospital, Nepal.," Kathmandu University medical journal (KUMJ), vol. 2, no. 3, pp. 188-192, 2004.

[24] K. L. Shrestha, N. D. Pant, R. Bhandari, S. Khatri, B. Shrestha, and B. Lekhak, "Re-emergence of the susceptibility of the Salmonella spp. isolated from blood samples to conventional first line antibiotics," Antimicrobial Resistance and Infection Control, vol. 5, no. 1, article no. 22, 2016.

[25] J. M. Easow, N. M. Joseph, B. A. Dhungel, B. Chapagain, and P. G. Shivananda, "Blood stream infections among febrile patients attending a teaching hospital in Western Region of Nepal," Australasian Medical Journal, vol. 3, no. 10, pp. 633-637, 2010.

[26] J. R. Andrews, K. Vaidya, C. Bern et al., "High Rates of Enteric Fever Diagnosis and Lower Burden of Culture-Confirmed Disease in Peri-urban and Rural Nepal," The Journal of Infectious Diseases, 2017.

[27] C. N. Thompson, S. D. Blacksell, D. H. Paris et al., "Undifferentiated febrile illness in Kathmandu, Nepal," The American Journal of Tropical Medicine and Hygiene, vol. 92, no. 4, pp. 875-878, 2015.

[28] D. Adhikari, D. Acharya, P. Shrestha, and R. Amatya, "Ciprofloxacin susceptibility of Salmonella enteric serovar Typhi and Paratyphi A from blood samples of suspected enteric fever patients," International Journal of Infection and Microbiology, vol. 1, no. 1, 2012.

[29] P. Pramod, L. Binod, A. Ritu, B. Sharad, and P. Prakash, "Enteric fever caused by Salmonella enterica serovar paratyphi A: An emerging health concern in Nepal," African Journal of Microbiology Research, vol. 10, no. 42, pp. 1784-1791, 2016.

[30] D. Acharya, D. R. Bhatta, S. Malla, S. P. Dumre, N. Adhikari, and B. P. Kandel, "Salmonella enterica serovar Paratyphi A: an emerging cause of febrile illness in Nepal.," Nepal Medical College journal : NMCJ, vol. 13, no. 2, pp. 69-73, 2011.

[31] R. M. Humphries, F. C. Fang, F. M. Aarestrup, and J. A. Hindler, "In vitro susceptibility testing of fluoroquinolone activity against salmonella: Recent changes to CLSI standards," Clinical Infectious Diseases, vol. 55, no. 8, pp. 1107-1113, 2012.
[32] T. Kadhiravan, N. Wig, A. Kapil, S. K. Kabra, K. Renuka, and A. Misra, "Clinical outcomes in typhoid fever: adverse impact of infection with nalidixic acid-resistant Salmonella typhi.," $B M C$ Infectious Diseases, vol. 5, article no. 37, 2005.

[33] G. A. Jacoby, "Mechanisms of resistance to quinolones," Clinical Infectious Diseases, vol. 41, supplement 2, pp. S120-S126, 2005.

[34] E. Giraud, A. Cloeckaert, D. Kerboeuf, and E. Chaslus-Dancla, "Evidence for active efflux as the primary mechanism of resistance to ciproffoxacin in Salmonella enterica serovar typhimurium," Antimicrobial Agents and Chemotherapy, vol. 44, no. 5, pp. 1223-1228, 2000.

[35] D. Acharya, S. Trakulsomboon, S. K. Madhup, and S. Korbsrisate, "Antibiotic susceptibility pattern and the indicator of decreased ciprofloxacin susceptibility of Salmonella enterica serovar Typhi isolated from Dhulikhel Hospital, Nepal.", Japanese Journal of Infectious Diseases, vol. 65, no. 3, pp. 264267, 2012.

[36] C. M. Parry, T. T. Hein, G. Dougan, N. J. White, and J. J. Farrar, "Typhoid fever," The New England Journal of Medicine, vol. 347, no. 22, pp. 1770-1782, 2002.

[37] E. J. Threlfall, L. R. Ward, B. Rowe et al., "Widespread occurrence of multiple drug-resistant Salmonella typhi in India," European Journal of Clinical Microbiology \& Infectious Diseases, vol. 11, no. 11, pp. 990-993, 1992.

[38] B. N. Harish and G. A. Menezes, "Antimicrobial resistance in typhoidal salmonellae," Indian Journal of Medical Microbiology, vol. 29, no. 3, pp. 223-229, 2011.

[39] Y. Kumar, A. Sharma, and K. R. Mani, "Re-emergence of susceptibility to conventionally used drugs among strains of Salmonella Typhi in central west India," The Journal of Infection in Developing Countries, vol. 5, no. 03, 2011.

[40] V. Lakshmi, R. Ashok, J. Susmita, and V. V. Shailaja, "Changing trends in the antibiograms of Salmonella isolates at a tertiary care hospital in Hyderabad," Indian Journal of Medical Microbiology, vol. 24, no. 1, pp. 45-48, 2006.

[41] S. Kariuki, M. A. Gordon, N. Feasey, and C. M. Parry, "Antimicrobial resistance and management of invasive Salmonella disease," Vaccine, vol. 33, no. 3, pp. C21-C29, 2015. 

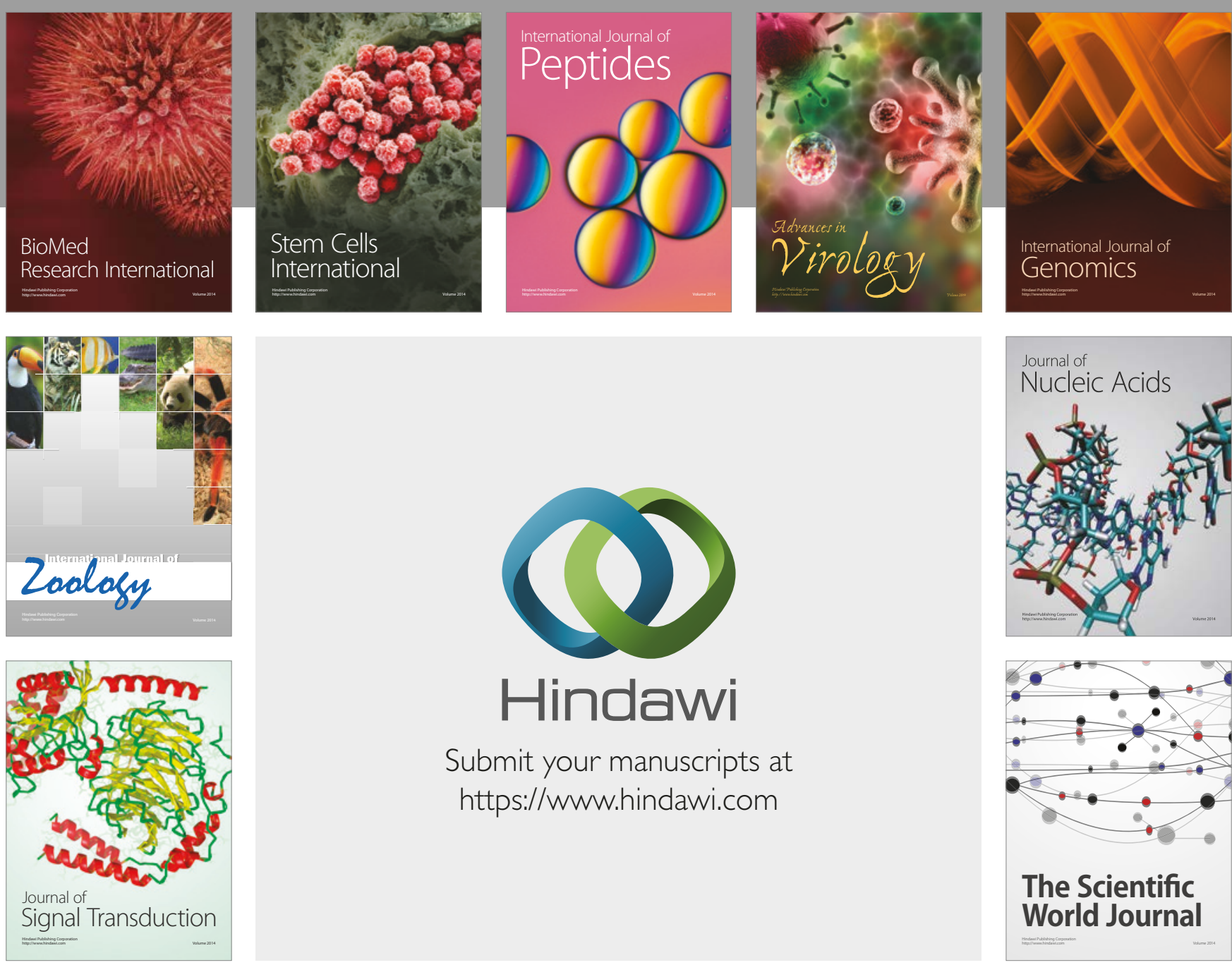

Submit your manuscripts at

https://www.hindawi.com
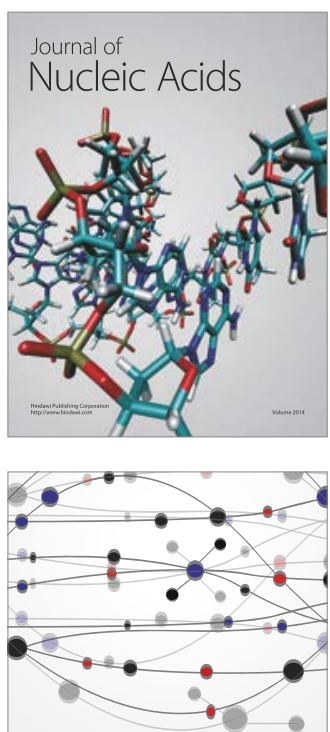

The Scientific World Journal

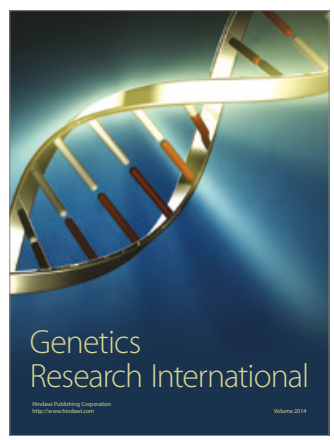

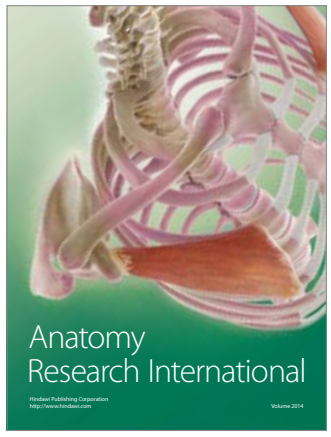

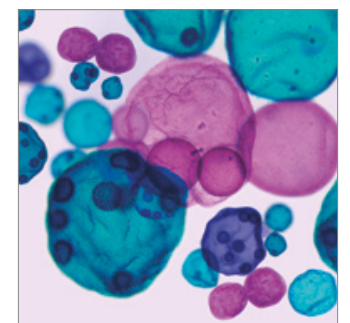

International Journal of Microbiology
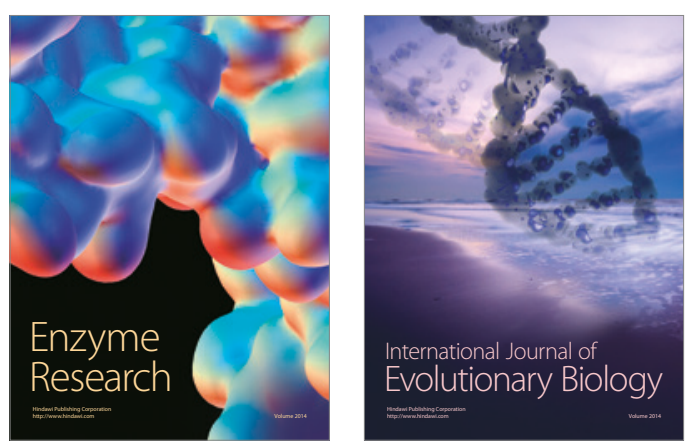
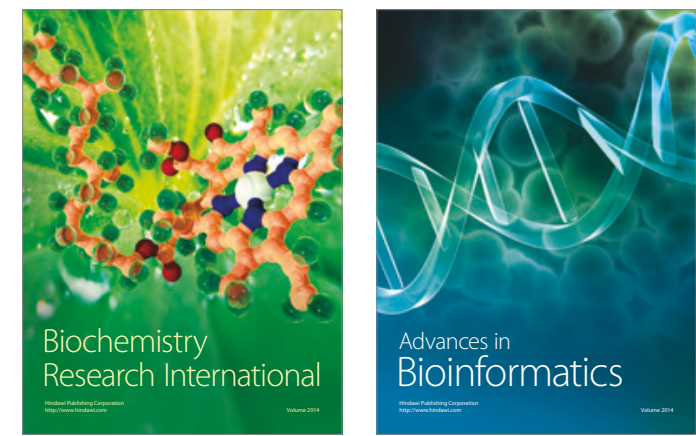

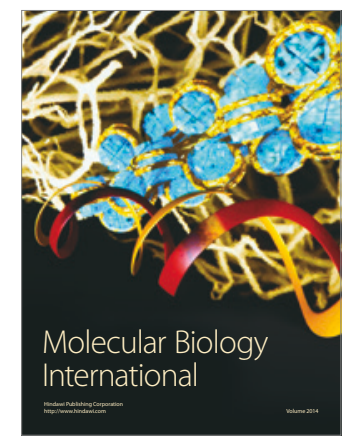

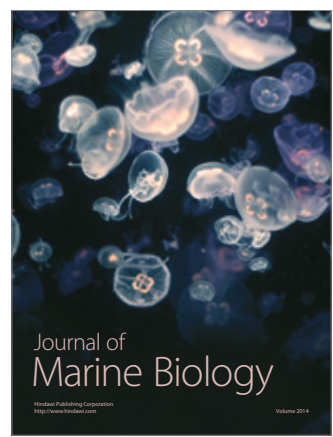

